\title{
Ant-behavior Inspired Intelligent NanoNet for Targeted Drug Delivery in Cancer Therapy
}

\author{
Lin Lin, Fupeng Huang, Hao Yan, Fuqiang Liu and Weisi Guo
}

\begin{abstract}
Targeted drug delivery system is believed as one of the most promising solutions for cancer treatment due to its lowdose requirement and less side effects. However, both passive targeting and active targeting rely on systemic blood circulation and diffusion, which is actually not the real "active" drug delivery. In this paper, an ant-behavior inspired nanonetwork composing of intelligent nanomachines is proposed. A big intelligent nanomachine take small intelligent nanomachines and drugs to the vicinity of of the tumor area. The small intelligent nanomachines can coordinate with each other to find the most effective path to the tumor cell for drug transportation. The framework and mechanism of this cooperative network are proposed. The route finding algorithm is presented. The convergence performance is analytically analyzed where the influence of the factors such as molecule degradation rate, home-destination distance, number of small nanomachines to the convergence is presented. Finally the simulation results validate the effectiveness of the proposed mechanism and analytical analysis.
\end{abstract}

Index Terms - ant, NanoNet, molecular communication, targeted drug delivery, cancer therapy

\section{INTRODUCTION}

Cancer, which involves the abnormal cell growth, is among the leading causes of death for years worldwide. Once it goes into stage IV with metastasis, cancer/tumor cells spread to other parts of body via lymphatic or blood vessels [2], then the mortality rate becomes significantly higher. Chemotherapy is a widely used cancer treatment method for many types of cancers, by which the anti-cancer drugs are introduced into the blood stream, address cancer, and stop cancer cell division by killing them [3]. The major drawbacks of chemotherapy include the inefficacy and the serious side-effect where the drugs not only kill cancer cells, but also kill other normal cells.

Targeted drug delivery is a method of delivering medication to specific parts of the body where there is solely diseased tissue, thereby avoiding interaction with healthy tissue [4]-[7]. In this way, the targeted delivery can improve efficacy while reducing side-effects. Targeted drug delivery is classified as

This work was supported in part by National Natural Science Foundation, China (61971314), in part by Science and Technology Commission of Shanghai Municipality (19510744900), in part by the EU Horizon 2020 Programme (792799), and in part by Natural Science Foundation of Shanghai (19ZR1426500). Part of this work has been presented at the 4th Workshop on Molecular Communications, Linz, Austria, 2019 [1]. Corresponding author: Weisi Guo.

L. Lin and F. Liu are wth the College of Electronics and Information Engineering, Tongji University, Shanghai, China

F. Huang and H. Yan are with the School of Electronic, Information and Electrical Engineering, Shanghai Jiao Tong University, China.

W. Guo is with Cranfield University, MK43 0AL, UK.

L. Lin is also with the School of Engineering, University of Warwick, UK. passive targeting and active targeting [8]. In passive targeting, by cloaking the drug-loaded nanoparticle with some sort of coating, the drugs are able to stay in circulation for a longer period of time. Some research might study the optimization issue of release pattern [9]. Active targeting of drug-loaded nanoparticles enhances the effects of passive targeting to make the nanoparticle more specific to a target site [10]. The examples include the use of cell-specific ligands or $\mathrm{pH}$ responsive materials [11].

We found that no matter passive targeting or active targeting, the movement of the drug-loaded nanoparticles is passive, relying on systemic blood circulation and diffusion, which makes the drug delivery less efficient and slow. It is expected that the nanorobot can be used for targeted drug delivery, which can actively move close to the target [12]. Several studies have been conducted to design nanorobots for drug delivery function. For example, a self-propelled polymer-based multilayer nanorobots with porous membrane was proposed for drug release [13]. A chemically powered nanomotor for nanoscale cargo delivery was proposed in [14]. A magnetic micromotor vehicle for transporting durg-loaded magnetic polymeric particles was proposed in [15]. Reference [16] proposed a ultrasound driven nanowire motors which could perform drug delivery by a light-triggered release. Those literature do propose mechanisms for active moving of nanorobots. However, how to control the nanorobot is still a challenge. Some papers did not mention the methods, and some other papers gave the control mechanisms but it is obviously difficult for implementation. Moreover, due to the limited size, at least in its early stage the nanorobot would be resource constraint with simple intelligence, and can only carry a tiny amount of drugs.

For the ant colony [17]-[19], each individual ant only has very limited resources and ability, but the groups of the ants can form remarkable collective intelligence. Whats more, by laying down molecules along the trail of the ants, an optimal trail to the destination can be established. Inspired by that, in this paper we propose a similar nanorobot network named NanoNet, for the targeted drug delivery in cancer therapy. The aim is that by the collaboration of the nanorobot/nanomachines via non-diffusive molecular communication [20]-[22], the formed NanoNet can intelligently find the best path to the tumor cells and transport drugs. Compared with our previous work [1], more details about the mechanism is presented, and a complete analysis and discussion is added. The major contributions of this paper include:

1) A complete framework of NanoNet for intelligent targeted drug delivery is proposed, which includes the drug 


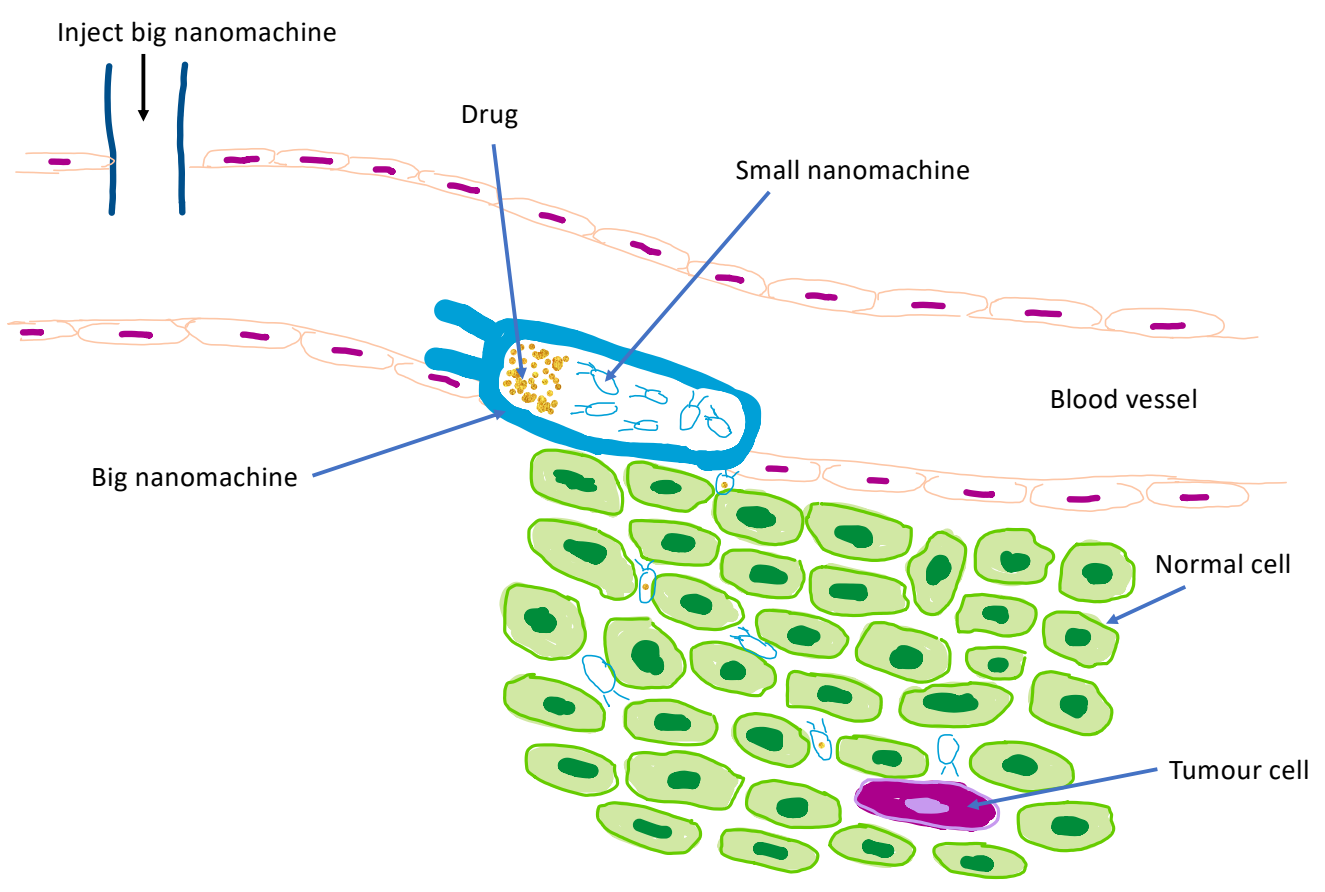

Fig. 1: Framework of NanoNet.

delivery along blood vessels or and through interstitial fluids.

2) Under the proposed NanoNet framework, the mechanism and algorithm for small nanomachines via non-diffusive molecular communication to find the destination, i.e., the tumor cell, is proposed and presented in detail.

3) The convergence performance for intelligent small nanomachines to find the best path for drug delivery is analytically analyzed, and the discussion is presented.

4) The effectiveness of the proposed mechanism and algorithm and the convergence conditions are evaluated by simulations.

The rest of the paper is organized as follows. In Section II, we present the framework and the system model. Section III presents the proposed mechanism of the collective ant behavior to active drug delivery. In Section IV, the convergence to the shortest path is analytically analyzed. The simulation results are provided in Section V. Finally, Section VI concludes the paper.

\section{Framework AND System Model}

The overall goal of NanoNet is to deliver drugs to the destination, i.e., the tumor cell. In the proposed framework, the entire delivery process is composed of two steps, as shown in Fig. 1. For the first step, a big nanomachine, which contains $M$ small nanomachines and drugs, moves in the blood vessel and is anchored on the blood vessel wall in the vicinity of the tumor cell. It is assumed that the big nanomachine is injected into the blood vessel as close to the tumor as possible. The position of the tumor could be found by existing techniques such as MRI or mobile nanosensors [23]. The tumor cells discrete more microvesicles, e.g., exosomes, than normal cells, which can be used as a sign for the possible existence of the tumor cells [24]. Therefore, the big nanomachine can stop and anchor itself when it senses abnormally high exosomes. For the second step, the small nanomachines carry drugs and start moving from the big nanomachine, which is named as "home", to the destination through interstitial fluids and extracellular fluid among cells [25], [26]. Because there exist specific detectable proteins on the surface of tumor cell [27], the small nanomachine can use this kind of proteins to locate the destination when they move. The small nanomachines go back to the home, carry drugs, move to the destination for drug delivery again.

The diameter of blood vessels varies for different parts of human body. It ranges from about 25 millimeters for the aorta to only 8 micrometers in the capillaries [28]. The extracellular space between cells ranges from several nanometers to tens of nanometers [29], [30]. So in this paper it is assumed that the size (diameter) of the big nanomachine is $6-8 \mu \mathrm{m}$, which is close to the size of red cells. It can go through blood vessels, but cannot go into the interstitial fluid from the blood vessel. It is assumed that the size (diameter) of the small nanomachine is around $10 \mathrm{~nm}$, to make sure it can go through most of extracellular space among cells. It is also assumed that the big nanomachine does not affect the normal propagation of blood in the blood vessels, including hindering the normal flow of blood cells or plasma, or forming clots close to the blood vessel walls.

Because the small nanomachines are more size constraint than the big nanomachine, and they need to coordinate with each other intelligently to find the best route between the home and the destination, which is much complicated, therefore, in this paper we focus on the mechanism of the second step.

The path model is described as a lattice graph in an 


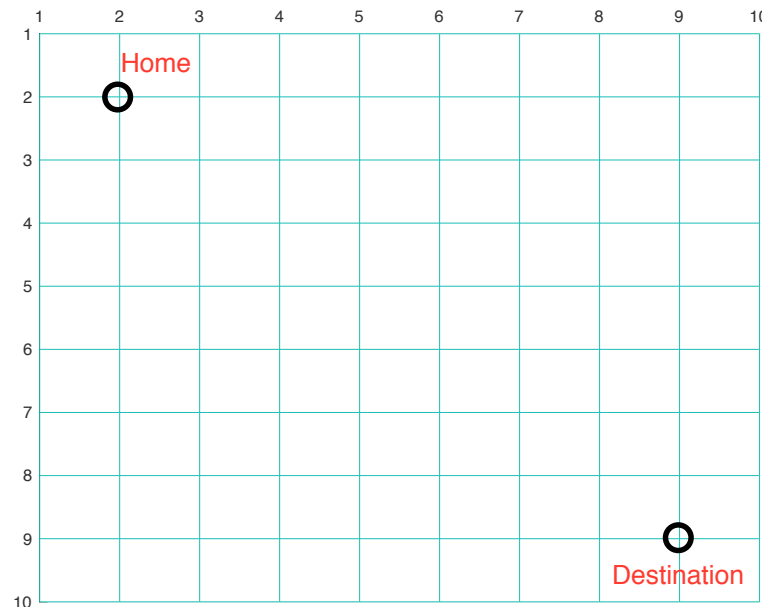

Fig. 2: An example of lattice graph representing the path model. The "home" is at $(2,2)$ and the "destination" is at $(9,9)$.

Euclidean space $\mathbf{R}^{2}$. An example is shown in Fig. 2. The grid is considered as a pathway among cells. The vertex is denoted by $(i, j)$, where $i$ and $j$ are the x-coordinate and $y$-coordinate. The physical distance between the adjacent vertices is assumed to be 1 , unit distance. Because the space beyond blood vessels is filled with interstitial fluids and extracellular matrix, which pose hurdles in the movements of the drug-carrying nanomachines, it is assumed that the edges between the adjacent vertices in the graph can have different weights. In the graph, it is assumed that there is one home with the location of $\left(x_{h}, y_{h}\right)$ and one destination with the location of $\left(x_{d}, y_{d}\right)$.

In the beginning, $M$ small nanomachines carrying drugs start to move from the home. The aim of these nanomachines is to arrive at the destination, e.g. tumor location, and release drugs there. Then the nanomachines go back to the home, load drugs again, and repeat the drug delivery process.

Because the small nanomachines are size constraint and resource constraint, they have very limited ability. They also do not know the path from home to destination in advance. Therefore, finding a path to the destination, especially, finding the optimal path to the destination, is a challenge.

It is assumed that the small nanomachines can release two types of adhesive molecules, named home molecules and destination molecules. These molecules are assumed to be non-diffusive, i.e., once they are released by nanomachines, they cannot diffuse. These kind of adhesive surface binding molecules exist widely in biology. The examples of the adhesive molecules include collagen, elastin, fibronectin and laminin [21], [31], [32]. Because the molecules are adhesive, they will almost not undergo thermal diffusion, and are less affected by the components in the interstitial fluid. In this paper, the system is assumed to be discrete both spatially and temporally. Spatially, the molecules can only be laid down at the vertices. Consecutive vertices are neighbors. Temporally, the unit of time is one iteration where each individual small nanomachine move one step from one vertex to its neighboring vertex.
For a specific location $(i, j)$, the concentration of home molecules after the $n$th iteration is denoted by $\phi_{i, j}(n)$. Similarly, the concentration of destination molecules at the location $(i, j)$ after the $n$th iteration is denoted by $\psi_{i, j}(n)$. The same types of molecules released by different nanomachines can be mixed together, and the total concentration is the sum of the molecular concentrations released by different nanomachines. Home molecules and destination molecules do not influence or react with each other.

It is assumed that the molecules degradate over time with degradation rate $\rho$ [33]. For example, if there is no newly laid down molecules, then from the $n$th iteration to $(n+1)$ th iteration, the concentration of home molecules can be expressed as

$$
\phi_{i, j}(n+1)=(1-\rho) \phi_{i, j}(n)-\eta,
$$

where $\eta$ is assumed to be a zero-mean Gaussian random variable. The degradation is very important for the small nanomachines to find the shortest path between home and destination. This will be discussed in the latter part of this paper in detail. It is also assumed that the nanomachines have the same moving speed, and they do not collide with each other when the nanomachines meet on the route.

\section{Proposed Mechanism AND Algorithm}

In this section, the mechanism of the collaboration of small nanomachines to find the route between home and destination is proposed.

In the beginning, $M$ small nanomachines carrying drugs start to move from the home at the same time. Initially they wander randomly on the lattice graph. The nanomachines release home molecules when they travel from home to destination. The home molecules will later be used by nanomachines to find the route from the destination to home. In our proposed scheme, the nanomachine releases $Q$ home molecules at home position, and releases $Q-q$ molecules after it moves one step away from home. Then after $n$ step movement, that nanomachine will release $Q-n q$ molecules at its location. If $Q-n q<0$, then the nanomachine will not release molecules. The reason that the nanomachine releases less home molecules as it goes away from the home is that it has less information of the home as it goes further away from the home. It has less confidence about where the home is. To be a by-product, a concentration gradient is formed based on the proposed molecule releasing rule. The nanomachine will find its way to home based on the positive gradient direction. The molecules are non-diffusive [20], [21] and degradate over time.

Once the nanomachines arrive at the destination, they unload the drugs, and go back to the home for the next round drug movement. The nanomachines do not move randomly as their first time travel from home to destination. As mentioned earlier, they will follow the positive gradient direction of home molecules along the path to go back to the home, provided that there are molecules on the path. It is possible that when the nanomachine goes from home to destination, before it arrives at the destination, $Q-n q$ already becomes negative. Then 
there is no molecule releasing for the nanomachine's following movement until it arrives the destination.

The rule for selecting next step movement for a nanomachine which is at certain vertex in the lattice graph is like this: assuming that the nanomachine has three possible options of moving direction for the next step, i.e., left, right, and front (sometimes it may have two possible options when it is on the edge of the graph. The logic of the rule is similar). Assuming that the real concentrations of the corresponding neighboring vertices are $C_{l}, C_{r}, C_{f}$, then the probabilities that the nanomachine move to left vertex, right vertex, and front vertex are expressed as

$$
\begin{gathered}
P_{l}=\frac{C_{l}}{\left(C_{l}+C_{r}+C_{f}\right)}, \\
P_{r}=\frac{C_{r}}{\left(C_{l}+C_{r}+C_{f}\right)}, \\
P_{f}=\frac{C_{f}}{\left(C_{l}+C_{r}+C_{f}\right)} .
\end{gathered}
$$

The nanomachine will choose one moving direction based on probabilities.

Similar to the movement from home to destination, the nanomachines release destination molecules when they move from destination to home. They also reduce the amount of molecules with the reducing speed $q$ molecules/step as they move.

The drug movement is performed repeatedly until the end.

It should be noted that the home molecules and destination molecules do not influence each other. They can co-exist without reaction. And the nanomachines have the ability to differentiate that two types of molecules and detect their concentrations within one-step distance, i.e., the position the nanomachine stands on and its neighboring positions on the graph.

After the $n$th round of iterations, the concentrations for home molecules and destination molecules at the location $(i, j)$ can be expressed as

$$
\begin{aligned}
& \phi_{i, j}(n)=(1-\rho) \phi_{i, j}(n-1)-\eta+\sum_{1}^{M} \tau_{i, j}^{m}(n), \\
& \psi_{i, j}(n)=(1-\rho) \psi_{i, j}(n-1)-\xi+\sum_{1}^{M} \delta_{i, j}^{m}(n) .
\end{aligned}
$$

where $\tau_{i, j}^{m}(n)$ and $\delta_{i, j}^{m}(n)$ represent the concentrations of home molecules and destination molecules released by nanomachine $m$ at location $(i, j)$ at the $n$th iteration. $\xi$ is the noise term for degradation of the destination molecules.

The algorithm for the route selection by small nanomachines is shown in Algorithm 1.

For the probability calculation in step 9, in the initial phase when the concentrations of the next possible positions are all zero, or in the middle phase, when the concentrations of the next possible positions are the same, then these positions in each direction calculation have equal probabilities.

Because the small nanomachines move between home and destination back and forth, they lay down molecule trails. A shorter path, would get marched over more frequently (the

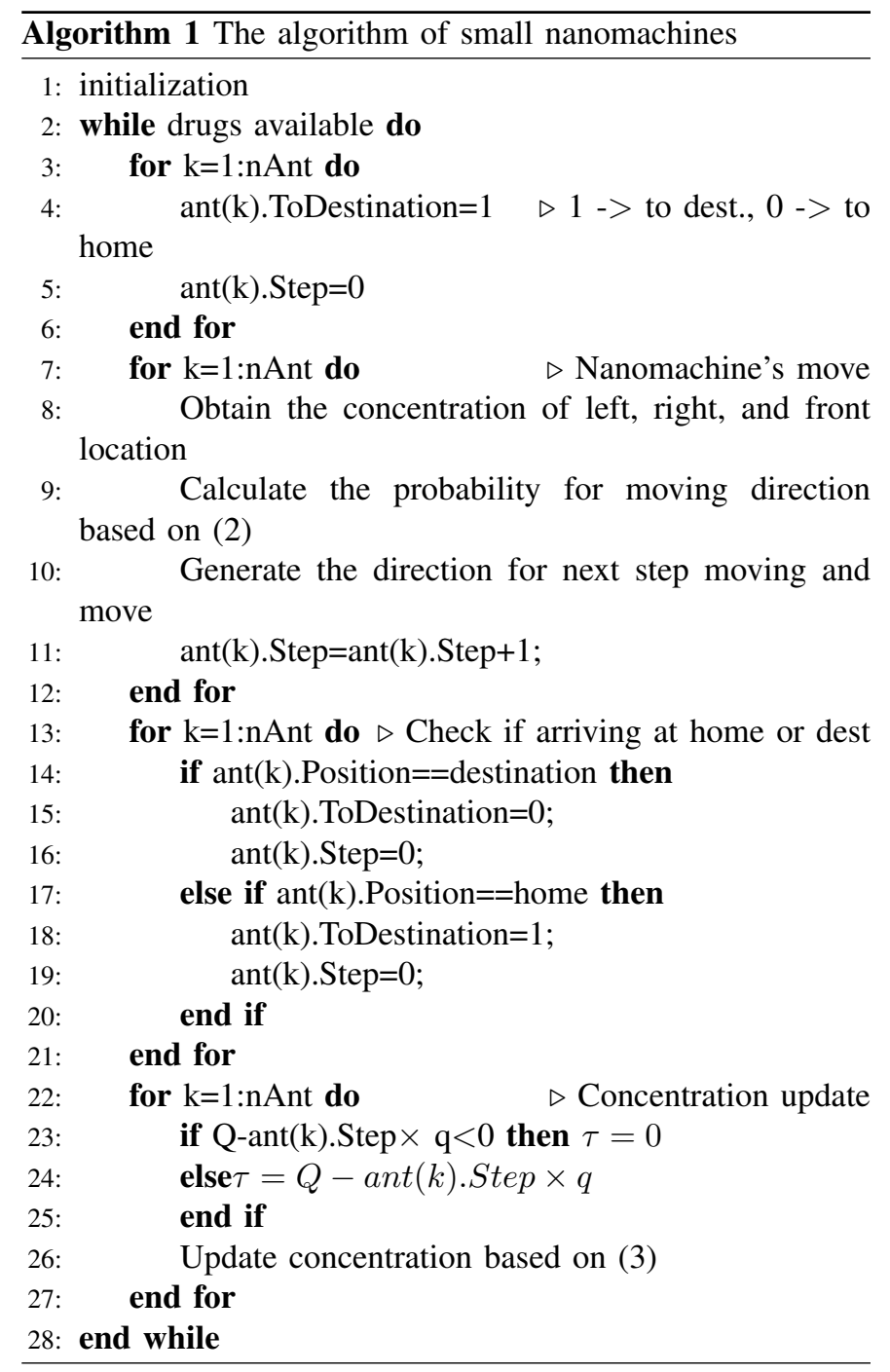

nanomachine which follows shorter path will come back earlier than others), and thus the molecule density becomes higher on shorter paths than longer ones. If other nanomachine find such a path, they are likely not to keep traveling at random, but instead to follow the trail, returning and reinforcing it if they eventually find the destination. The positive feedback eventually leads to the nanomachines following a shortest path. The next section will give analytical analysis and convergence discussion.

\section{Analytical Analysis and Convergence DISCUSSION}

The proposed mechanism in Section III actually forms an optimization problem to find the shortest path by distributed computing as

$$
\underset{\forall \text { path } \in \mathbb{P}}{\operatorname{argmin}} d(\text { path }),
$$

where $d$ (path) is the distance for certain path and $\mathbb{P}$ is the space of all possible paths. The small nanomachines are autonomous computational entities and they communicate by molecular communication via the released non-diffusive molecules. 
Next, the conditions for the convergence is discussed. There are three levels regarding the convergence in our scenario: as time goes to infinity, 1) no single path is formed; 2) a single path is formed, but not the shortest path between home and destination; 3) a single path is formed, which is the shortest path (optimal solution) between home and destination. These three conditions correspond to not converge, converge to some path but not the shortest path, and converge to the shortest path.

Proposition 1 The path formed by the nanomachines will not converge if $\rho \geq \frac{q}{Q} \geq \frac{1}{N-1}$ holds, where $N$ is the length of the shortest path.

Proof As mentioned in Section III, $Q$ is the amount of molecules a single nanomachine releases when it is at home or destination before it starts moving to the other end. $q$ is the difference of the amount of molecules the nanomachine releases for one step moving. For example, a nanomachine which starts at home towards destination, releases $Q$ molecules at the home location, and after one step movement, it releases $Q-q$ molecules. after $n$ step movement, that nanomachine will release $Q-n q$ molecules at its location.

If the nanomachine can go from the destination to home following the trails of molecules along the positive concentration gradient direction, two conditions must be satisfied: 1) the shortest distance between the home and the destination cannot be too long that there is no molecule released by the nanomachine along the path from the home to the destination, 2) the degradation rate cannot be too fast that the correct molecular concentration gradient towards the home or destination does not exist.

For the first condition, $Q-(N-1) q$ is the amount of molecules released by the nanomachine which follows the shortest path from home to destination just one step before arriving at the end. The following should hold

$$
Q-(N-1) q>0,
$$

so that when the nanomachine returns, it has molecule trail information to follow. Therefore, the condition that the path will not converge can be expressed as

$$
Q-(N-1) q \leq 0
$$

We rewrite it as

$$
\frac{q}{Q} \geq \frac{1}{N-1} .
$$

For the second condition, when the nanomachine moves after $n$ step movement from home or destination, it will release $Q-n q$ molecules. After it moves one more step and arrives at the $(n+1)$ th location, it will release $Q-(n+1) q$ molecules. At the same time, molecules at the last location (the location it arrives after the $n$th step) degradatete with rate $\rho$, so the concentration becomes $(1-\rho)(Q-n q)$. The convergence may be achieved if

$$
(1-\rho)(Q-n q)>Q-(n+1) q,
$$

is satisfied, i.e., the molecular concentration of the location closer to the origin (home or destination) is greater than that of the neighboring location further to the origin. Then we can have

$$
\rho<\frac{q}{Q-n q}, \quad n=0,1, \ldots, N .
$$

Equation (9) has to be satisfied for all possible values of $n$. The minimum value of $\frac{q}{Q-n q}$ is obtained when $n=0$. Therefore, the convergence condition becomes

$$
\rho<\frac{q}{Q}
$$

Then not converge condition can be expressed as

$$
\rho \geq \frac{q}{Q}
$$

Combing (7) and (11), the condition that the path formed by the nanomachines will not converge is

$$
\rho \geq \frac{q}{Q} \geq \frac{1}{N-1} .
$$

It should be noted that (12) is the condition that the path does not converge, but it does not mean that it will converge once the complement of (12) is satisfied.

Next, we would like to discuss when the precondition

$$
0<\rho<\frac{q}{Q}<\frac{1}{N-1}
$$

is satisfied, what else condition should be satisfied for the convergence to optimal solution (shortest path).

Let us consider the scenario with only one nanomachine in the environment first, i.e., $M=1$. We define Self-shortest path as below.

Definition 1 Self-shortest path is defined as a path where there are no two separate points (at least one point in the middle) in the path, which are the same vertex or the neighboring vertices on the lattice graph.

An example is shown in Fig. 3. An example of the selfshortest path is shown in (a). If a small nanomachine goes from $(1,2)$ to $(6,2)$ as the red line in $(a)$, at the same time laying down tracking molecules, then it has only one route, the same route, to go back by following the tracking molecules. An example of a path which is not a self-shortest path in shown in (b). This is because the vertex $(3,2)$ and $(4,2)$, which are separate points on the path as defined, are neighboring vertices. If a small nanomachine goes from $(1,2)$ to $(6,2)$ as the red line in (b), at the same time laying down tracking molecules, then when it goes back to $(1,2)$, it would follow the red route shown in (c) because of the gradient.

Two cases will be discussed below:

Case 1: the first round path of the nanomachine from home to destination is a self-shortest path. In this case, according to the rule selection in (2), the return path from destination to home will be the same as the path from home to destination, and the nanomachine will follow this path forever. This first round path from home to destination is totally random, and has a very low probability to be the shortest path.

Case 2: the first round path of the nanomachine from home to destination is not a self-shortest path. In this case, when 


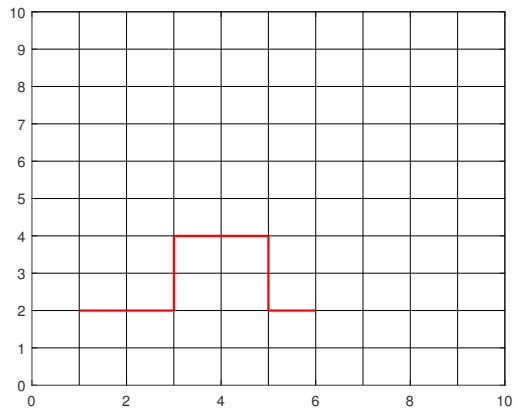

(a) An example of the self-shortest path.

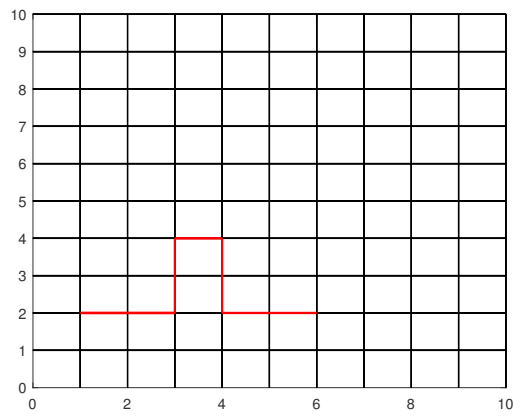

(b) An example of a path which is not a self-shortest path.

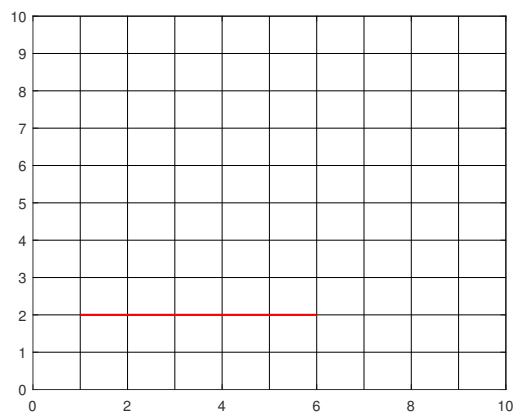

(c) The shortest path if a small nanomachine's route is the red line in (b).

Fig. 3: Explanation of the self-shortest path. (a) An example of the self-shortest path. (b) An example of a path which is not a self-shortest path in shown. (c) The shortest path if a small nanomachine's route is the red line in (b).

the nanomachine moves back from the destination to home, it will more likely choose shorter path at the point which makes the first round path from home to destination not selfshortest. The shorter path will be obviously different from the first round path from home to destination. The reason the shorter path will be chosen is that when the nanomachine faces two direction options, the concentration from the shorter path's point is larger than that from the longer path's point. This is because of the mechanism of gradually reducing release of molecules as the nanomachine moves. After that, the probability the nanomachine chooses the shorter path increases for the following back and forth between home and destination. As time goes to infinity, the nanomachine will follow the shorter path in probability. This can be expressed as

$$
\lim _{i->+\infty} \operatorname{Pr}\left(\text { Step }_{\text {htod }}=R\right)=1, \quad R \geq N,
$$

where $i$ is the number of iteration, $\operatorname{Pr}()$ represents probability, and $S t e p_{\text {htod }}$ is the number of steps from home to destination, $R$ is a value greater than or equal to $N$. The final path in Case 2 has higher probability to be the shortest path than in Case 1.

A short proof of convergence to a single path in probability is given below.

It is assumed that there are two possible paths the nanomachine can walk through, the length of path1 is shorter than that of path2, denoted by path $1<$ path2. The concentration of the first movement into path1 is denoted as $C_{p 1}$, and the concentration of the first movement into path2 is denoted as $C_{p 2}$. There is no constraint if the concentration of the first movement into path1 is larger than that of path2 or not. The nanomachine will choose to go via path1 or path2 with the probability $\frac{C_{p 1}}{C_{p 1}+C_{p 2}}$ and $\frac{C_{p 2}}{C_{p 1}+C_{p 2}}$. The ratio is $\frac{C_{p 1}}{C_{p 2}}$.

If path1=path2, then the amounts of molecules released at the end of path1 and path2 are the same. Considering the entry probability of path1 and path2, the ratio of the amounts of molecules released at the end of path1 and path2, denoted as $\frac{C_{p 1}^{\prime}}{C_{p 2}^{\prime}}$, will be the same as $\frac{C_{p 1}}{C_{p 2}}$. However, path2 is assumed to be longer than path1, so the amounts of molecules released at the end of path2 will be smaller than that of path1, and then we have

$$
\frac{C_{p 1}^{\prime}}{C_{p 2}^{\prime}}>\frac{C_{p 1}}{C_{p 2}} .
$$

This means that the nanomachine will have more chance to go back from path1 than from path2. As the nanomachines go back and forth between home and destination, there will be more and more molecules laying down on path1. Finally, the convergence is achieved.

Next, we discuss the scenario where more than one nanomachine are in the environment. More nanomachines will increase the solution space for the first round movement from home to destination. More nanomachines will also accelerate the convergence for the whole NanoNet.

No matter one nanomachine scenario or multiple nanomachine scenario, the first round movement of the nanomachine from home to destination is the key factor for the convergence. It determines the solution space. If this solution space contains the optimal solution, then the shortest path will be achieved in probability as time goes to infinity. If the solution space after the first round movement from home to destination does not contain the optimal solution, then the shortest path will never be explored. An example is shown in Fig. 4. In that figure, assuming a nanomachine goes from one red point to another for the first round movement. The blue and red points represent the whole solution space on which the nanomachine goes. We can easily find that the solution space does not include a shortest path between the two red points.

If the number of nanomachines goes to infinity, then the probability that the first round movement contains the shortest 


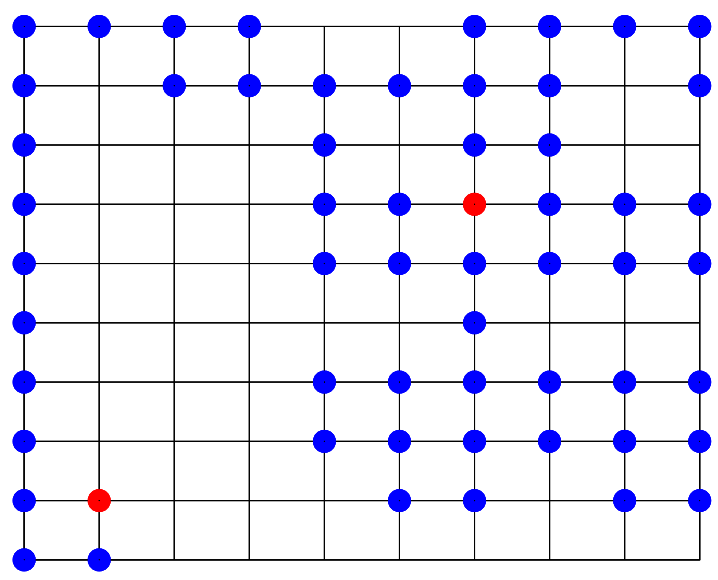

Fig. 4: An example showing that a solution space does not include optimal solution.

TABLE I: Simulation Parameters

\begin{tabular}{cl}
\hline Parameters & Value \\
\hline Lattice graph range & $10 \times 10$ \\
Shortest path length $N$ & $3-15$ \\
Maximum molecule release $Q$ & $N-1-100$ \\
Number of small nanomachines M & $1-50$ \\
degradation rate $\rho$ & $0-0.9$ \\
Decremental released molecules $q$ & 1 \\
\hline
\end{tabular}

path tends to 1. Then as time goes to infinity, the whole network will converge to the shortest path in probability, which can be expressed as

$$
\lim _{i->+\infty} \operatorname{Pr}\left(\operatorname{Step}_{\text {htod }}(m)=N\right)=1, \quad m=1,2, \ldots, M
$$

The convergence is also related to our proposed mechanism. In our proposed mechanism, if the nanomachine does not go through certain vertex in the graph, then according to (2), it will have no chance to go through that vertex later. Then it may lose the chance to achieve the shortest path. But if the mechanism can give a small probability for the nanomachines to go into that vertex even if that vertex is not passed by the nanomachine, then the convergence to the shortest path will definitely happen.

\section{Simulation Results}

In this section, simulations are performed by MATLAB to validate the effectiveness of the proposed mechanism as well as the analytical analysis and discussion. The conditions for the convergence rely on several factors, such as molecule degradation rate, home-destination distance, the number of nanomachines, etc. We will discuss the influence of those factors to the convergence performance.

A $10 \times 10$ lattice graph is used. In the simulations, the position of the home and destination is determined first. The shortest path length is set to $10-15$. The maximum molecule release $Q$ is set to $N$ to 100 . The number of small nanomachines is set to 1 to 50 . Then for the fixed number of small nanomachines, the simulation experiments are run 100 times. For each time, no more than 10000 iterations is conducted. The iterations end if stable convergence is seen. The degradation rate is set between 0 and 0.9. Simulation parameters are shown in Table 1.

Fig. 5 shows the molecule concentration summation of the two kinds of molecules on the trails for different iterations and different degradation rates for a single simulation run. The degradation rate $\rho$ is set as $0.01,0.02$, and 0.03 . These values are determined under the condition in (13). It can be seen that after a number of iterations, the single path is formed clearly, which is the shortest path. It should also be noted that even with the same shortest path length, the path itself may be different. We can also see that when $\rho=0.03$, after 700 iterations, the path converges. But when $\rho=0.02$, after 700 iterations, the path does not converge. when $\rho=0.02$, after 2000 iterations, the path converges. But when $\rho=0.01$, after 3000 iterations, the path does not converge.

This phenomena can also be reflected in Fig. 6. In Fig. 6, it can be seen that all the three curves finally converge to a stable value. The curve with larger $\rho$ converges faster than the other two curves with relatively small $\rho$ value. The reason is because if $\rho$ is larger, then the concentration on the longer path will degradate faster. It help accelerating the convergence. If $\rho$ is smaller, for example the solid blue curve, it can be seen that it takes nearly 8000 iterations to converge. Because of the route selection rule in (2), even if the nanomachine goes into the shorter path, it will still go into longer path. This is because the molecule concentration of the vertices on the longer path still has a relatively high value.

Fig. 7 shows Iterations versus degradation rate for different home-destination distances. It can be seen that as the increase of the home-destination distance, the iteration for the convergence increases. This is because the nanomachines need more movement from home to destination or from destination to home. It can also be seen that the increase of $\rho$ leads to less iterations for convergence. The reason is that bigger $\rho$ means the trailing molecules degradate faster, then the molecules on the less passed route will degradate more quickly and the shortest path trail will be established more quickly. Then the less iterations are needed for the convergence.

Fig. 8 shows the iteration versus number of small nanomachines for different home-destination distances. We can conclude from the figure that the increase of the number of small nanomachines leads to faster convergence. The reason might be: 1) more nanomachines have more chance to explore the solution space in the first round movement from home to destination. This may lead to smaller iterations to achieve convergence. 2) more nanomachines will release more molecules during their movement between the home and destination. This will accelerate the convergence. It can be also seen that after the number of the small nanomachines reaches 30 , the iterations becomes stable. This reflects that the influence of reason 2 to the convergence is not as significant as reason 1 .

Fig. 9 shows the maximum molecule release $Q$ versus home-destination distance $N$. This result comes from (13). $Q$ 


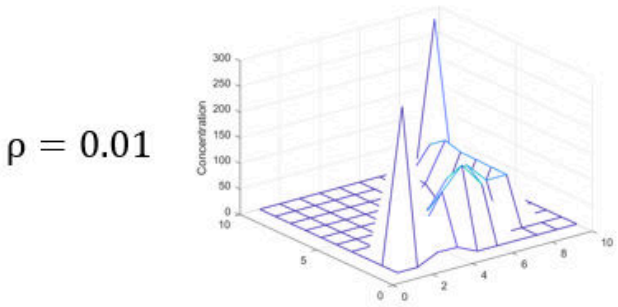

(a) 1500 Iterations

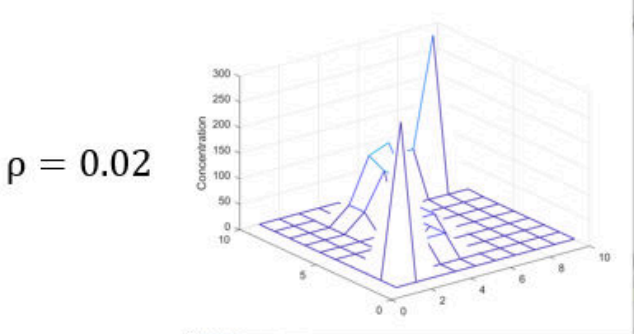

(d) 500 Iterations

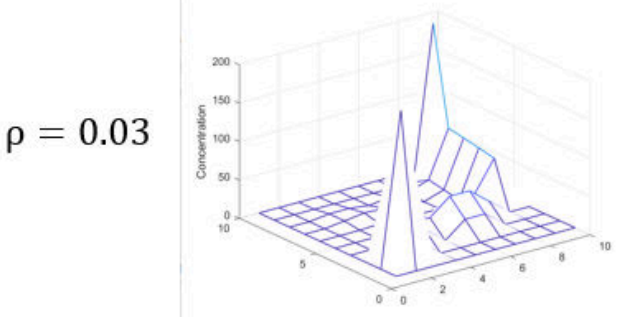

(g) 300 Iterations

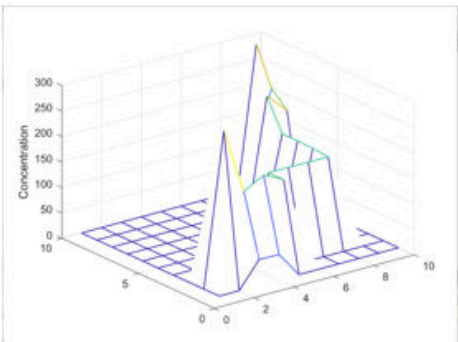

(b) 3000 Iterations

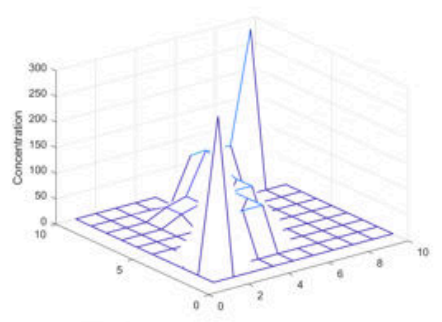

(e) 700 Iterations

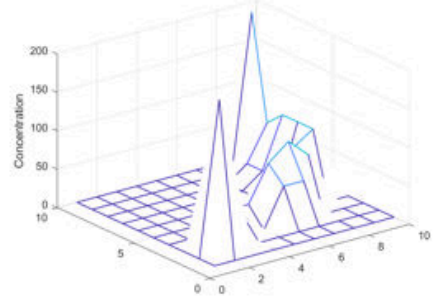

(h) 500 Iterations

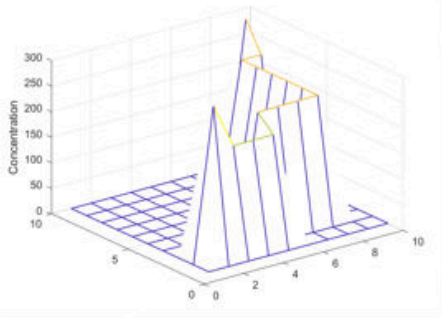

(c) 9000 Iterations

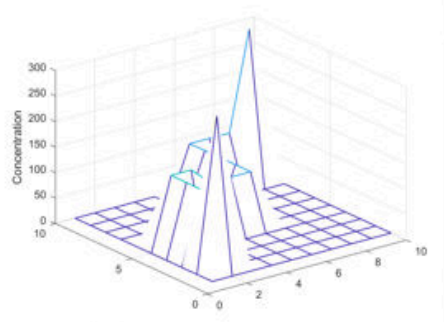

(f) 2000 Iterations

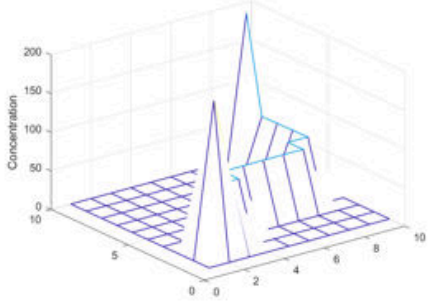

(i) 700 Iterations

Fig. 5: Molecule concentration on lattice graph for different iterations and different degradation rates.

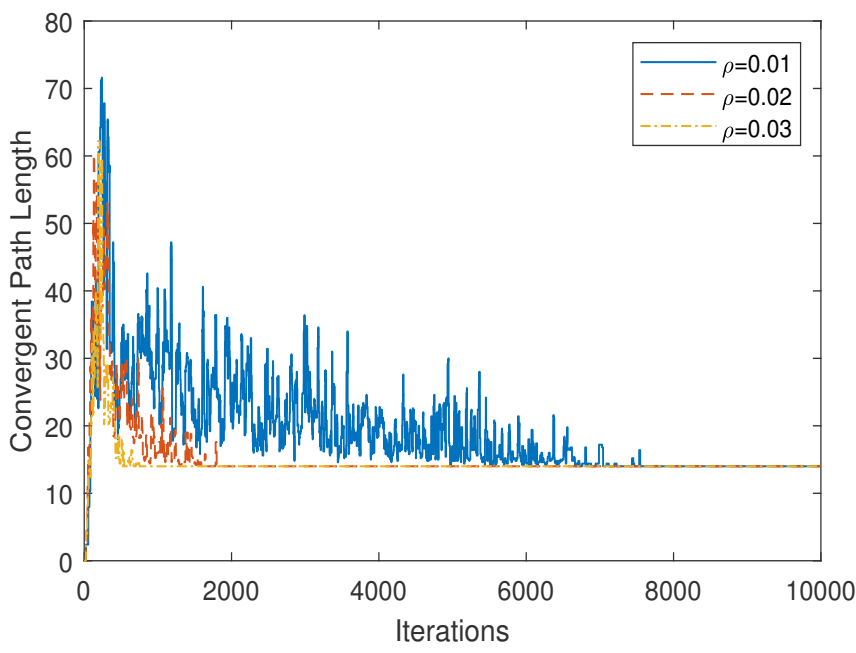

Fig. 6: Convergence length versus iterations: an example.

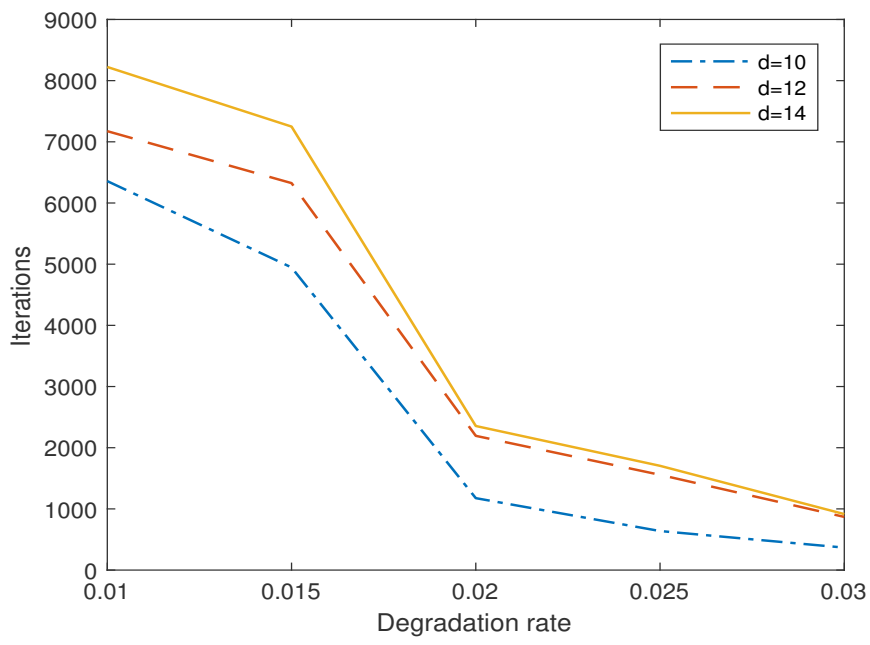

Fig. 7: Iterations versus degradation rate for different homedestination distances.

\section{CONCLUSION}

needs to be large enough so that there exist molecules on the shortest trail between the home and destination. In this figure, the upper left area with respect to the curve is the possible convergence region.
In this paper, an ant-behavior inspired intelligent NanoNet is proposed for targeted drug delivery system. It is a real active drug delivery method, because it does not only rely on blood vessel circulation. A complete framework for the 


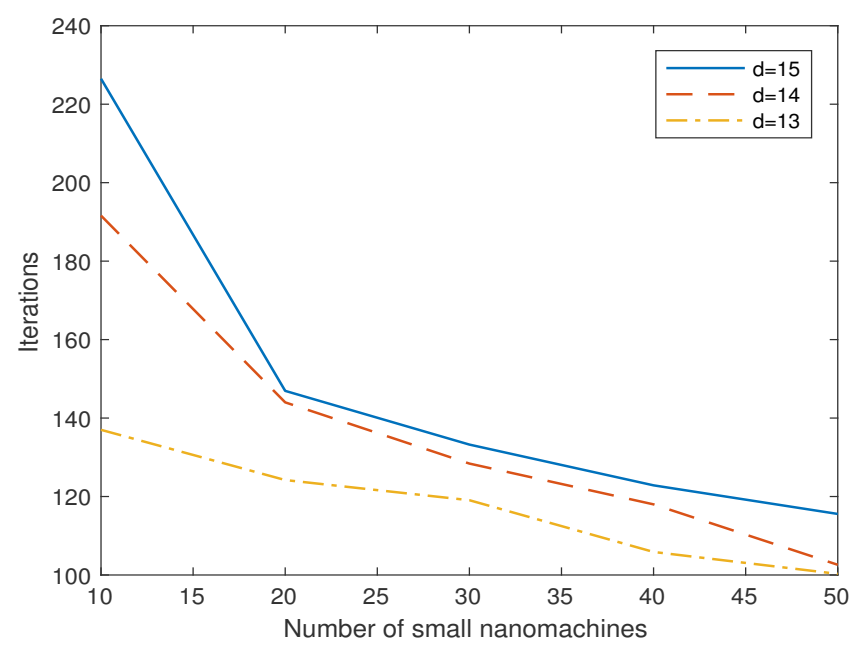

Fig. 8: Iteration versus number of small nanomachines for different home-destination distances.

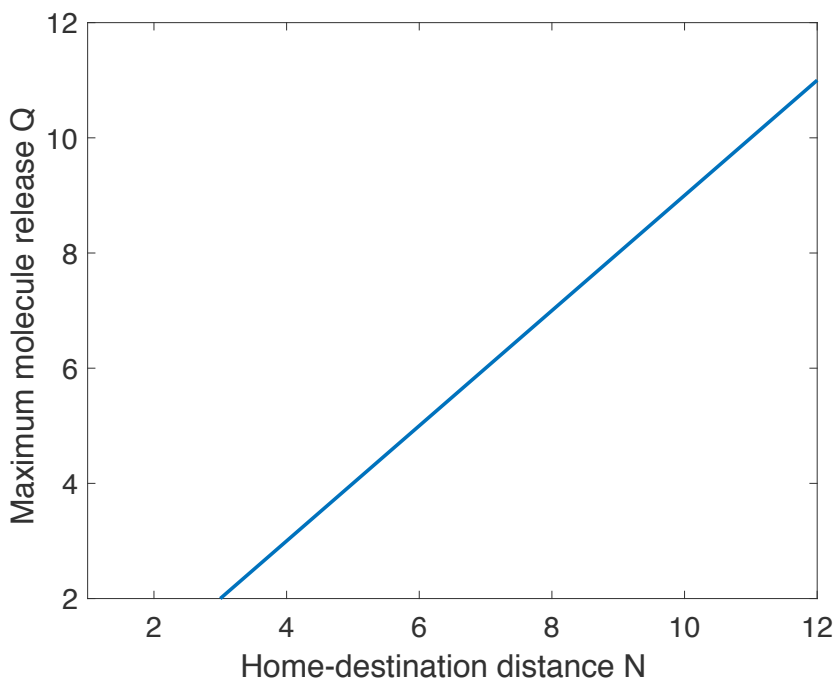

Fig. 9: Maximum molecule release $Q$ versus home-destination distance $N$.

whole system is proposed. With the collaboration of simple intelligence, the path between the home and destination can be found. The mechanism and algorithm are presented in detail. The convergence performance is analytically discussed and validated by simulations. The influence of key factors such as molecule degradation rate to the convergence performance is evaluated. Future work would take different edge weights of the graph into account and analyze the corresponding convergence performance.

\section{REFERENCES}

[1] L. Lin, H. Yan, F. Liu, and W. Guo, "Ant-behavior inspired intelligent nanonet for targeted drug delivery in cancer therapy," in 4th Workshop on Molecular Communications, Linz, Austria, 2019.

[2] G. P. Gupta and J. Massagué, "Cancer metastasis: building a framework," Cell, vol. 127, no. 4, pp. 679-695, 2006.
[3] T. Iwamoto, "Clinical application of drug delivery systems in cancer chemotherapy: review of the efficacy and side effects of approved drugs," Biological and pharmaceutical bulletin, vol. 36, no. 5, pp. 715-718, 2013.

[4] U. A. Chude-Okonkwo, R. Malekian, B. T. Maharaj, and A. V. Vasilakos, "Molecular communication and nanonetwork for targeted drug delivery: A survey," IEEE Commun. Surveys Tuts., vol. 19, no. 4, pp. 3046-3096, Fourth Quarter 2017.

[5] Y. Chahibi, "Molecular communication for drug delivery systems: A survey," Nano Commun. Netw., vol. 11, pp. 90-102, 2017.

[6] Y. Chahibi, I. F. Akyildiz, S. Balasubramaniam, and Y. Koucheryavy, "Molecular communication modeling of antibody-mediated drug delivery systems," IEEE Trans. Biomed. Eng., vol. 62, no. 7, pp. 1683-1695, Jul. 2015.

[7] M. Femminella, G. Reali, and A. V. Vasilakos, "A molecular communications model for drug delivery," IEEE Trans. Nanobiosci., vol. 14, no. 8, pp. 935-945, 2015.

[8] N. Bertrand, J. Wu, X. Xu, N. Kamaly, and O. C. Farokhzad, "Cancer nanotechnology: the impact of passive and active targeting in the era of modern cancer biology," Advanced drug delivery reviews, vol. 66, pp. $2-25,2014$.

[9] T. N. Cao, A. Ahmadzadeh, V. Jamali, W. Wicke, and R. Schober, "Diffusive mobile mc for controlled-release drug delivery with absorbing receiver," in 2019 IEEE Int. Conf. Commun. (ICC), May 2019.

[10] F. Marcucci and F. Lefoulon, "Active targeting with particulate drug carriers in tumor therapy: fundamentals and recent progress," Drug discovery today, vol. 9, no. 5, pp. 219-228, 2004.

[11] J. D. Byrne, T. Betancourt, and L. Brannon-Peppas, "Active targeting schemes for nanoparticle systems in cancer therapeutics," Advanced drug delivery reviews, vol. 60, no. 15, pp. 1615-1626, 2008.

[12] C. Mavroidis and A. Ferreira, "Nanorobotics: past, present, and future," in Nanorobotics. Springer, 2013, pp. 3-27.

[13] Z. Wu, Y. Wu, W. He, X. Lin, J. Sun, and Q. He, "Self-propelled polymer-based multilayer nanorockets for transportation and drug release," Angewandte Chemie International Edition, vol. 52, no. 27, pp. 7000-7003, 2013.

[14] X. Ma, K. Hahn, and S. Sanchez, "Catalytic mesoporous janus nanomotors for active cargo delivery," Journal of the American Chemical Society, vol. 137, no. 15, pp. 4976-4979, 2015.

[15] W. Gao, D. Kagan, O. S. Pak, C. Clawson, S. Campuzano, E. ChuluunErdene, E. Shipton, E. E. Fullerton, L. Zhang, E. Lauga et al., "Cargotowing fuel-free magnetic nanoswimmers for targeted drug delivery," small, vol. 8, no. 3, pp. 460-467, 2012.

[16] V. Garcia-Gradilla, S. Sattayasamitsathit, F. Soto, F. Kuralay, C. Yardımcı, D. Wiitala, M. Galarnyk, and J. Wang, "Ultrasoundpropelled nanoporous gold wire for efficient drug loading and release," Small, vol. 10, no. 20, pp. 4154-4159, 2014.

[17] M. Dorigo, M. Birattari, C. Blum, M. Clerc, T. Stützle, and A. Winfield, Ant Colony Optimization and Swarm Intelligence: 6th International Conference, ANTS 2008, Brussels, Belgium, September 22-24, 2008, Proceedings. Springer, 2008, vol. 5217.

[18] T. Stutzle and M. Dorigo, "A short convergence proof for a class of ant colony optimization algorithms," IEEE Trans.Evol. Comput., vol. 6, no. 4 , pp. 358-365, 2002.

[19] M. Dorigo and M. Birattari, Ant colony optimization. Springer, 2010.

[20] T. Nakano, Y. Okaie, S. Kobayashi, T. Koujin, C.-H. Chan, Y.-H. Hsu, T. Obuchi, T. Hara, Y. Hiraoka, and T. Haraguchi, "Performance evaluation of leader-follower-based mobile molecular communication networks for target detection applications," IEEE Trans. Commun., vol. 65, no. 2, pp. 663-676, Feb. 2017.

[21] T. Obuchi, Y. Okaie, T. Nakano, T. Hara, and S. Nishio, "Inbody mobile bionanosensor networks through non-diffusion-based molecular communication," in Proc. IEEE ICC. IEEE, 2015, pp. 1078-1084.

[22] N. Farsad, H. B. Yilmaz, A. Eckford, C. B. Chae, and W. Guo, "A comprehensive survey of recent advancements in molecular communication," IEEE Commun. Surveys Tuts., vol. 18, no. 3, pp. 1887-1919, Third Quarter 2016.

[23] R. Mosayebi, A. Ahmadzadeh, W. Wicke, V. Jamali, R. Schober, and M. Nasiri-Kenari, "Early cancer detection in blood vessels using mobile nanosensors," IEEE Trans. Nanobiosci., vol. 18, no. 2, pp. 103-116, 2018.

[24] J. Skog, T. Würdinger, S. Van Rijn, D. H. Meijer, L. Gainche, W. T. Curry Jr, B. S. Carter, A. M. Krichevsky, and X. O. Breakefield, "Glioblastoma microvesicles transport rna and proteins that promote tumour growth and provide diagnostic biomarkers," Nature cell biology, vol. 10, no. 12, p. 1470, 2008. 
[25] P. M. Gullino, S. H. Clark, and F. H. Grantham, "The interstitial fluid of solid tumors," Cancer Research, vol. 24, no. 5, pp. 780-797, 1964.

[26] S. Salehi, N. S. Moayedian, S. H. Javanmard, and E. Alarcón, "Lifetime improvement of a multiple transmitter local drug delivery system based on diffusive molecular communication," IEEE Trans. Nanobiosci. vol. 17, no. 3, pp. 352-360, 2018.

[27] B. K. Shin, H. Wang, A. M. Yim, F. Le Naour, F. Brichory, J. H. Jang, R. Zhao, E. Puravs, J. Tra, C. W. Michael et al., "Global profiling of the cell surface proteome of cancer cells uncovers an abundance of proteins with chaperone function," Journal of Biological Chemistry, vol. 278, no. 9, pp. 7607-7616, 2003.

[28] T. Lin, T. Smith, and C. Pinnock, Fundamentals of anaesthesia. Cambridge University Press, 2016.

[29] J. Scallan, V. H. Huxley, and R. J. Korthuis, "Capillary fluid exchange: regulation, functions, and pathology," in Colloquium Lectures on Integrated Systems Physiology-From Molecules to Function, vol. 2, no. 1. Morgan \& Claypool Publishers, 2010, pp. 1-94.

[30] A. Pietak and M. Levin, "Exploring instructive physiological signaling with the bioelectric tissue simulation engine," Frontiers in bioengineering and biotechnology, vol. 4, p. 55, 2016.

[31] T. Nakano, Y. Okaie, S. Kobayashi, T. Koujin, C.-H. Chan, Y.-H Hsu, T. Obuchi, T. Hara, Y. Hiraoka, and T. Haraguchi, "Performance evaluation of leader-follower-based mobile molecular communication networks for target detection applications," IEEE Trans. Commun., vol. 65, no. 2, pp. 663-676, 2016.

[32] S. Iwasaki, J. Yang, and T. Nakano, "A mathematical model of non-diffusion-based mobile molecular communication networks," IEEE Commun. Lett., vol. 21, no. 9, pp. 1969-1972, 2017.

[33] A. Ahmadzadeh, H. Arjmandi, A. Burkovski, and R. Schober, "Comprehensive reactive receiver modeling for diffusive molecular communication systems: Reversible binding, molecule degradation, and finite number of receptors," IEEE Trans. Nanobiosci., vol. 15, no. 7, pp. 713727, Oct. 2016 
2020-04-01

\section{Ant-behavior inspired intelligent nanonet for targeted drug delivery in cancer therapy}

Lin, Lin

IEEE

Lin L, Huang F, Yan H, et al., (2020) Ant-behavior inspired intelligent nanonet for targeted drug delivery in cancer therapy. IEEE Transactions on NanoBioscience, Volume 19, Issue 3, July 2020, pp. 323-332

https://doi.org/10.1109/TNB.2020.2984940

Downloaded from Cranfield Library Services E-Repository 